Research Article

\title{
On the Geometry of the Movements of Particles in a Hamilton Space
}

\author{
A. Ceylan Coken ${ }^{1}$ and Ismet Ayhan ${ }^{2}$ \\ ${ }^{1}$ Faculty of Art and Sciences, Department of Mathematics, Suleyman Demirel University, 32260 Isparta, Turkey \\ ${ }^{2}$ Faculty of Education, Department of Mathematics Education, Pamukkale University, 20070 Denizli, Turkey \\ Correspondence should be addressed to A. Ceylan Coken; ceylancoken@sdu.edu.tr
}

Received 31 December 2012; Accepted 15 February 2013

Academic Editor: Abdelghani Bellouquid

Copyright (c) 2013 A. C. Coken and I. Ayhan. This is an open access article distributed under the Creative Commons Attribution License, which permits unrestricted use, distribution, and reproduction in any medium, provided the original work is properly cited.

We studied on the differential geometry of the Hamilton space including trajectories of the motion of particles exposed to gravitational fields and the cotangent bundle.

\section{Introduction}

As known, a Hamilton space is constructed as a differentiable manifold and a real valuable function defined on its cotangent bundle. The second order partial differentiation (Hessian) of this real valuable function with respect to momentum coordinate $\left(p_{i}\right)$ determines a metric tensor on the cotangent bundle. However, Hessian of the Hamiltonian with respect to momentum coordinate determines a metric tensor on the manifold. This metric tensor is considered by Miron [1]. Recently, many studies have been done on the metrics defined on the cotangent bundles, and most of these studies are on two distinguished metrics. One of these metrics is the Riemann extension of the torsion-free affine connection [2-4] and the other one is the diagonal lift in cotangent bundle $[1,5]$. Willmore [3] showed that a torsion-free affine connection on a manifold determines canonically a pseudoRiemannian metric on the cotangent bundle. Furthermore, he expressed this pseudo-Riemannian metric as the Riemann extension of the affine connection. Akbulut et al. [5] defined a diagonal lift of a Riemannian metric of a manifold to its cotangent bundle, and they studied the differential geometry of the cotangent bundle with respect to this Riemann metric. Oproiu [6] studied the differential geometry of tangent bundle of a Lagrange manifold when this tangent bundle is endowed with pseudo-Riemannian metric obtained from fundamental tensor field by a method similar to the obtaining of the complete lift of a pseudo-Riemannian metric on a differentiable manifold. Ayhan [7, 8] obtained the images on the cotangent bundle of the some tensor fields (i.e., functions, vector fields, and 1-forms, and tensor fields with types $(1,1)$, $(0,2)$ and $(2,0))$ on the tangent bundle of a Lagrange manifold which are obtained by vertical, complete, and horizontal lifts under the Legendre transformation.

In this paper, it is proved that the trajectories of particles exposed to gravitational fields are geodesics and the Hamilton function as represented of the total energy of system is constant along these trajectories. We studied the differential geometry of the cotangent bundle $T^{*} M$ of the Hamilton space $M$ including the trajectories of particles exposed to gravitational fields. We obtained that the pseudo-Riemannian metric $G$ on $T^{*} M$ corresponds to pseudo-Riemannian metric $g^{C}$ on $T M$ with respect to Legendre transformation, and we showed that $G$ is the Riemann extension of the Levi-Civita connection. Moreover we considered an almost product structure $P$ is defined on $T^{*} M$. By means of $P$ and $G$, an almost symplectic structure $\theta$ on $T^{*} M$ is defined. Finally we obtained that the coefficients of the Levi-Civita connection $\dot{\nabla}$ and Riemann curvature tensor $K$ of $\left(T^{*} M, G\right)$ and we found the condition under which $T^{*} M$ is locally flat.

In this study, all the manifolds and the geometric objects are assumed to be $C^{\infty}$, and we use the Einstein summation convention. 


\section{The Movement in a Hamilton Space}

The fundamental physical concept is that a gravitational field is identical to geometry of the Hamilton space. This geometry is determined by Hamiltonian

$$
H\left(x^{i}, p^{i}\right)=\frac{1}{2} g^{i k}(x) p_{i} p_{k}
$$

where $g^{i k}(x)$ is a tensor with type $(2,0)$ given by $g^{i k} g_{k j}=$ $\delta_{j}^{i} \cdot g_{k j}(x)$ is local components of a (pseudo)Riemann metric tensor [9]. At the same time, the second order partial differentiation (Hessian) of Hamiltonian given by (1) with respect to momentum coordinate $\left(p_{i}\right)$ is equal to the following tensor type of $(2,0)$ :

$$
g^{i k}(x)=\frac{\partial^{2} H}{\partial p_{i} \partial p_{k}} .
$$

The Hamilton space $M$, called a Hamilton mechanic system by mechanists, consists of $n$-dimensional differentiable manifold $M$ and regular Hamiltonian $H$ given by (2) providing $\operatorname{det}\left[g^{i k}\right] \neq 0[1,10]$. The motion of every particle in the Hamilton space depending on time is represented as a curve $\gamma: I \subset R \rightarrow M$. For any time $t$, the position coordinates of every particle in the Hamilton space are given by $x^{i} \circ \gamma(t), i=1, \ldots, n$, or briefly $x^{i}, i=1, \ldots, n$, and, respectively, the velocity and momentum coordinates are given by $y^{i}=d x^{i} / d t, i=1, \ldots, n$, and $p_{i}=g_{i j} y^{j}, i=1, \ldots, n$.

The movement equation of any particle in the Hamilton space from the position $\left(x^{1}\left(t_{1}\right), \ldots, x^{n}\left(t_{1}\right)\right)$ to $\left(x^{1}\left(t_{2}\right), \ldots, x^{n}\left(t_{2}\right)\right)$ is determined by the canonic Hamilton equation which is defined by

$$
\frac{d x^{i}}{d t}=\frac{\partial H}{\partial p_{i}} ; \quad \frac{d p_{i}}{d t}=-\frac{\partial H}{\partial x^{i}} .
$$

The solution curves of the differential equation system in (3) are one-parameter group of diffeomorphisms of the Hamilton space [11]. The Hamiltonian $H$ is fixed on family of oneparameter curves, which defines a conservation law. As any particle is moving on any curve in the Hamilton space, the total energy $H$ of the system is the same on every point of curve. In other words, the Hamiltonian is not changed with respect to variable $t$ and the total energy $H$ must be constant as the particles move. Since the tangent vector field of a curve $C: t \rightarrow\left(x^{i}(t), p_{i}(t)\right), i=1, \ldots, n$, satisfies the canonic Hamilton equation in (3), this tangent vector field of $C$ is called the Hamilton vector field. Integral curves of the Hamilton vector field correspond to geodesics in the Hamilton space $M$ [12]. In Section 3, we proved that the integral curves of the Hamilton vector field on $T^{*} M$ correspond to geodesics on $M$ and also the value of Hamiltonian $H$ does not change on geodesics of $M$ for the Hamiltonian of the gravitational fields given by (1).

The Hamilton space $M$ is an $n$-dimensional differentiable manifold with $\left(U, x^{i}\right), i=1, \ldots, n$, the local chart and $T^{*} M$ is $2 n$-dimensional its cotangent bundle with $\left(\pi^{-1}(U), x^{i}, p_{i}\right), i=$ $1, \ldots, n$, the local chart, where $\pi: T^{*} M \rightarrow M$ is canonical projection, $x^{i}=x^{i}$ o $\pi$ and $p_{i}$ are the vector space coordinates of an element from $\pi^{-1}(U)$ with respect to the local frame $\left(d x^{1}, \ldots, d x^{n}\right)$ of $T^{*} M$ defined by the local chart $\left(U, x^{i}\right)$. In classical mechanics, $T^{*} M$ and $T M$ are called momentum phase space and velocity phase space, respectively. The tangent bundle of $T^{*} M$ has an integrable vector subbundle $V T^{*} M=\operatorname{Ker} \pi_{*}$ called the vertical distribution on $T^{*} M$. A nonlinear connection on $T^{*} M$ is defined by the horizontal distribution by $H T^{*} M$ and $H T^{*} M$ is complementary to $V T^{*} M$ in $T T^{*} M$. Thus $T T^{*} M=V T^{*} M \oplus H T^{*} M$. The system of the local vector fields $\left(\partial / \partial p_{1}, \ldots, \partial / \partial p_{n}\right)$ is a local frame in $V T^{*} M$ and the system of the local vector fields $\left(\delta / \delta x^{1}, \ldots, \delta / \delta x^{n}\right)$ is a local frame in $H T^{*} M$.

The Legendre transformation $\varphi$ is a diffeomorphism between the open set of $\widetilde{U} \subset T M$ and the open set of $U \subset T^{*} M$. Let $\left\{\delta / \delta x^{i}, \partial / \partial y^{i}\right\},\left\{d x^{i}, \delta y^{i}\right\}$ be an adapted frame (coframe) on $T M$ and $\left\{\delta / \delta x^{i}, \partial / \partial p_{i}\right\},\left\{d x^{i}, \delta p_{i}\right\}$ be an adapted frame (coframe) on $T^{*} M$. Then the differential geometric objects on $T^{*} M$ can be expressed in terms of those of TM by using the Legendre transformation as follows:

$$
\begin{gathered}
\left(\varphi^{-1}\right)^{*}\left(d x^{i}\right)=d x^{i}, \\
\left(\varphi^{-1}\right)^{*}\left(\delta y^{i}\right)=g^{i j} \delta p_{j}, \quad\left(\varphi^{-1}\right)^{*}\left(g_{i j}\right)=g_{i j} .
\end{gathered}
$$

\section{The Integral Curves and Metrics}

In this section, we studied the relation between the integral curves of the Hamilton vector field on $T^{*} M$ and the geodesics on $M$. Then we obtained the pseudo-Riemann metric $G$ on $T^{*} M$ by using two different methods. In addition, we defined an almost symplectic structure $\theta$ on $T^{*} M$ by using $G$ and an almost product structure $P$. Finally, the fact that the total energy $H$ is constant for each stage of the system as the system with $n$-particles moves with the effect of the gravitational field is reexpressed in terms of differential geometric objects on the cotangent bundle $T^{*} M$ of the Hamilton space $M$.

Theorem 1. Let $H$ be the Hamiltonian given by (1). Let $C$ be a curve in $T^{*} M, \gamma$ be a projection of $C$ to $M$; that is, $\pi \circ C=\gamma$, and let $w$ be a 1 -form associated with the tangent vector of curve $\gamma(t)$.

(i) If the curve $C$ is an integral curve of the Hamilton vector field $V$, the curve $\gamma$ is geodesic.

(ii) The Hamiltonian of the gravitational field $H$ is constant along the geodesic of the Hamilton space.

Proof. (i) Let $V$ be the Hamilton vector field. $V$ has local expression with respect to induced coordinate system on $T^{*} M$

$$
V=\frac{\partial H}{\partial p_{i}} \frac{\partial}{\partial x^{i}}-\frac{\partial H}{\partial x^{i}} \frac{\partial}{\partial p_{i}} .
$$

The tangent vector field of $C$ in $T^{*} M$ has local coordinate expression

$$
C_{*}\left(\frac{d}{d t}\right)=\frac{d x^{i}(t)}{d t} \frac{\partial}{\partial x^{i}}+\frac{d p_{i}(t)}{d t} \frac{\partial}{\partial p_{i}}
$$


with respect to induced coordinate system of $T^{*} M$. If the curve $C$ is an integral curve of the Hamilton vector field $V$, the equation $C_{*}(d / d t)=V_{C(t)}$ holds. From this equation, we obtain the following canonic Hamilton equations:

$$
\frac{d x^{i}(t)}{d t}=\frac{\partial H}{\partial p_{i}}=g^{i j} p_{j}, \quad \frac{d p_{i}(t)}{d t}=-\frac{\partial H}{\partial x^{i}} .
$$

The right part of the above equations is expressed by

$$
\frac{d}{d t}\left(g_{i j} \frac{\partial H}{\partial p_{j}}\right)+\frac{\partial H}{\partial x^{i}}=0 .
$$

Using the composite function differentiation, we get

$$
\frac{d p_{k}}{d t}+\frac{\partial}{\partial x^{k}}\left(g_{i j} \frac{\partial H}{\partial p_{j}}\right) \frac{d x^{k}}{d t}+\frac{\partial H}{\partial x^{i}}=0,
$$

and by

$$
\frac{d x^{k}}{d t}=g^{k a} p_{a}
$$

we get

$$
\frac{d p_{k}}{d t}+\frac{\partial}{\partial x^{k}}\left(g_{i j} \frac{\partial H}{\partial p_{j}}\right) g^{k a} p_{a}+\frac{\partial H}{\partial x^{i}}=0 .
$$

Next, transvecting by $g_{k a}$, we get

$$
g_{k a} \frac{d p_{k}}{d t}+S_{j}(x, p)=0,
$$

where

$$
S_{j}(x, p)=\frac{\partial}{\partial x^{k}}\left(g_{i j} \frac{\partial H}{\partial p_{j}}\right) p_{a}+g_{k a} \frac{\partial H}{\partial x^{i}} .
$$

We get a nonlinear connection on $T^{*} M$ defined by

$$
N_{j k}=S_{j}^{a}=\frac{1}{2} g_{k a} \frac{\partial^{2} H}{\partial x^{j} \partial p_{a}},
$$

where

$$
N_{j k}=S_{j}^{a}=\frac{1}{2} \frac{\partial S_{j}}{\partial p_{a}} .
$$

Then, we obtain

$$
\frac{\partial N_{j k}}{\partial p_{b}}=\frac{1}{2} g_{k a} \frac{\partial g^{a b}}{\partial x^{j}},
$$

and since the following equation is satisfied:

$$
\frac{\partial g^{a b}}{\partial x^{j}}=\frac{\partial}{\partial x^{j}} g\left(d x^{a}, d x^{b}\right),
$$

we get

$$
\frac{\partial N_{j k}}{\partial p_{b}}=-\Gamma_{j k}^{b}
$$

Subsequently we obtained that $N_{j k}=-\Gamma_{j k}^{b} p_{b}$ and $S_{j}(x, p)=$ $-\Gamma_{j k}^{b} p_{b} p_{a}$. If we substitute the above equation into (12), we get

$$
g_{k a} \frac{d p_{k}}{d t}-\Gamma_{j k}^{b} p_{b} p_{a}=0,
$$

and transvecting by $g^{k a}$, we get

$$
\frac{d p_{k}}{d t}-\Gamma_{j k}^{b} p_{b} g^{k a} p_{a}=0 .
$$

Thus,

$$
\frac{d p_{k}}{d t}-\Gamma_{j k}^{b} p_{b} \frac{d x^{k}}{d t}=0
$$

Then we get

$$
\nabla_{\left(d x^{k} / d t\right)\left(\partial / \partial x^{k}\right)} p_{j} d x^{j}=0 .
$$

Since $w=p_{i} d x^{i}$ is a 1 -form associated with the tangent vector, $\dot{\gamma}=d \gamma(t) / d t$ of curves $\gamma(t)$, and Riemann connection $\nabla$ satisfies the following property:

$$
\nabla_{\dot{\gamma}}(w(\dot{\gamma}))=2 g\left(\dot{\gamma}, \nabla_{\dot{\gamma}} \dot{\gamma}\right) \quad \text { for } w(\dot{\gamma})=g(\dot{\gamma}, \dot{\gamma}),
$$

we get

$$
g\left(\dot{\gamma}, \nabla_{\dot{\gamma}} \dot{\gamma}\right)=0 .
$$

Therefore it can be seen that straightforward the curve $\gamma(t)$ is a geodesic curve.

(ii) It is sufficient to show the Hamiltonian $H$ is not changed with respect to variable $t$ in order to prove the theorem. We calculate

$$
\frac{d H}{d t}=\frac{\partial H}{\partial x^{i}} \frac{d x^{i}}{d t}+\frac{\partial H}{\partial p_{i}} \frac{d p_{i}}{d t} .
$$

If we take into account (3), we obtain $d H / d t=0$. Thus $H$ is not dependent on value $t$.

Therefore, we obtain that the trajectories of particles exposed to gravitational fields are geodesics and the Hamilton function represented of the total energy of system is constant along these trajectories.

We consider differential geometric objects on the cotangent bundle $T^{*} M$ of the Hamilton space. Let us start by obtaining a metric on $T^{*} M$. A pseudo-Riemann metric $G$ on the cotangent bundle $T^{*} M$ of the Hamilton space is obtained by using two different ways. Firstly, the pseudo-Riemann metric on the cotangent bundle $T^{*} M$ is obtained as we were inspired by the paper of Willmore [3] as follows.

Theorem 2. The Levi-Civita connection $\nabla$ on $M$ determines canonically a pseudo-Riemannian metric on $T^{*} M$.

Proof. Let $P$ be a point on $T^{*} M$ such that $C(0)=P$. Let $X$ be a tangent vector to $C$ at $P$. The image of the curve $C(t)$ under the bundle projection map $\pi$ is a curve $\gamma(t)$ on $M$, passing through $p=\pi(P) \in U$. The curve $C(t)$ can be 
regarded as a field of covariant vectors $\omega(t)$ defined along the curve $\gamma(t)$. The covariant derivative $\left(\nabla_{d \pi(X)} \omega(t)\right)_{t=0}$ is a covector at $p$ which can be evaluated on the projected tangent vector $d \pi(X)$. This defines a quadratic differential form $Q$ on $T\left(T^{*} M\right)$. From this we obtain a bilinear form $G$ on $T^{*} M$ at $P$ by the usual formula

$$
G(X, Y)=Q(X+Y, X+Y)-Q(X, X)-Q(Y, Y),
$$

where $X$ and $Y$ are tangent vectors to $T^{*} M$ at $P$. We shall consider that $G$ corresponds to Riemann extension of $\nabla$ on $M$. We choose a local coordinate system $\left(x^{i}\right), i=1, \ldots, n$, valid in some neighborhood $U$ around $p$. Then a local coordinate system for $\pi^{-1}(U)$ is $\left(x^{i}, p_{j}\right)$, where $\omega=p_{j} d x^{j}$. The curve may be expressed locally by $t \rightarrow\left(x^{i}(t), p_{j}(t)\right)$ and the corresponding curve $\gamma$ is $t \rightarrow\left(x^{i}(t)\right)$. The vector $X$ at $P$ is given by $\left(\dot{x}^{i}(0), \dot{p}_{i}(0)\right)$ and its projection $d \pi(X)$ by $\dot{x}^{i}(0)$. Then, at when $t=0$, we have

$$
\begin{aligned}
\nabla_{d \pi(X)} \omega(t) & =\dot{x}^{i}(0)\left(\nabla_{i} p_{j}(t)\right) d x^{j} \\
& =\left[\frac{d p_{j}}{d t}-\Gamma_{i j}^{k} p_{k} \dot{x}^{i}(0)\right] d x^{j},
\end{aligned}
$$

where $\Gamma_{i j}^{k}$ are the connection coefficients of $\nabla$. We evaluate this covector on $d \pi(X)$ to get the number

$$
\begin{aligned}
Q(X, X) & =\left(\nabla_{d \pi(X)} \omega(t)\right)(d \pi(X)) \\
& =-\Gamma_{i j}^{k} p_{k} \dot{x}^{i} \dot{x}^{j}+\dot{p}_{j} \dot{x}^{j} .
\end{aligned}
$$

If the equation which is obtained above taken into account in (26), we get for $G(X, X)$ the following:

$$
\begin{aligned}
G(X, X) & =Q(2 X, 2 X)-2 Q(X, X) \\
& =2 Q(X, X) \\
& =-2 \Gamma_{i j}^{k} p_{k} \dot{x}^{i} \dot{x}^{j}+2 \dot{p}_{j} \dot{x}^{j} \\
& =\left(-2 \Gamma_{i j}^{k} p_{k} d x^{i} d x^{j}+2 d p_{j} d x^{j}\right)(X, X) .
\end{aligned}
$$

Therefore $G$ has local expression

$$
G=-2 \Gamma_{i j}^{k} p_{k} d x^{i} d x^{j}+2 d p_{j} d x^{j}
$$

with respect to the induced coordinates $\left(x^{i}, p_{i}\right)$ in $\pi^{-1}(U)$. Since the adapted dual frame on $T^{*} M$ is $\left(d x^{i}, \delta p_{i}\right)$, where

$$
\delta p_{i}=d p_{i}-p_{k} \Gamma_{i j}^{k} d x^{j}
$$

we get $G=2 \delta p_{i} d x^{j}$.

Secondly, the pseudo-Riemann metric on the cotangent bundle $T^{*} M$ is obtained as follows.

Theorem 3. Let $M$ be a manifold with a (pseudo)Riemann metric $g$. Then the pseudo-Riemannian metric $g^{C}$ on TM corresponds to the pseudo-Riemannian metric $G=2 \delta p_{i} d x^{k}$ on $T^{*} M$.
Proof. Let $g$ be a (pseudo)Riemannian metric on $M$ then $g^{C}$ given by $g^{C}=2 g_{j k} \delta y^{j} d x^{k}$ is a pseudo-Riemann metric on $T M$. By using the equalities in (4), we get

$$
\begin{aligned}
\left(\varphi^{-1}\right)^{*} & \left(g^{C}\right) \\
& =2\left(\varphi^{-1}\right)^{*}\left(g_{j k}\right)\left(\varphi^{-1}\right)^{*}\left(\delta y^{j}\right)\left(\varphi^{-1}\right)^{*}\left(d x^{k}\right) \\
& =2 \underbrace{g_{j k} g^{j i}}_{\delta_{k}^{i}} \delta p_{i} d x^{k},
\end{aligned}
$$

which gives a pseudo-Riemann metric $G=2 \delta p_{i} d x^{k}$ on $T^{*} M$.

In order to understand the relation between the pseudoRiemann manifold $\left(T^{*} M, G\right)$ and the symplectic manifold $\left(T^{*} M, \theta\right)$, we need to define an almost symplectic structure $\theta$ on the cotangent bundle $T^{*} M$ of the Hamilton space and an almost product structure $P$ on $T^{*} M$. The definition of $P$ and $\theta$ was obtained as we were inspired by the studies of Miron [10] for the Hamilton space.

Definition 4. Let $w=p_{i} d x^{i}$ be globally defined as 1-form on $T^{*} M$. The exterior differential $d w$ of the 1 -form $w$ is called an almost symplectic structure $\theta$ on the cotangent bundle $T^{*} M$ of the Hamilton space given by

$$
\theta=d w=\delta p_{i} \wedge d x^{i}
$$

Definition 5. Let $T^{*} M$ be a $2 n$-dimensional manifold. A mixed tensor field defines an endomorphism on each tangent space of $T^{*} M$. If there exists a mixed tensor field $P$ which satisfies

$$
P \circ P=I,
$$

we say that the field gives an almost product structure to $T^{*} M$.

We can consider the tensor field with type $(1,1)$ on $T^{*} M$ :

$$
P=\frac{\delta}{\delta x^{i}} \otimes d x^{j}-\frac{\partial}{\partial p_{i}} \otimes \delta p_{j} .
$$

Theorem 6. $P$ is an almost product structure on $T^{*} M$.

Proof. We have

$$
P\left(\frac{\delta}{\delta x^{\mathrm{i}}}\right)=\frac{\delta}{\delta x^{\mathrm{i}}}, \quad P\left(\frac{\partial}{\partial p_{i}}\right)=-\frac{\partial}{\partial p_{i}}
$$

from which $P \circ P=I$.

Theorem 7. Let $G$ be a pseudo-Riemann metric defined as Riemann extension of $\nabla$ in $M$ and let $P$ be an almost product structure on $T^{*} M . \theta$ is an almost symplectic structure associated with $(G, P)$. Nondegenerate skew-symmetric 2-form $\theta$ on $T^{*} M$ is given by following equation:

$$
\theta(X, Y)=G(P X, Y) \text {. }
$$


Proof. By using (33), the value of the vector fields $X, Y$ on $T^{*} M$ under $\theta$ is

$$
\begin{aligned}
\theta\left(X^{V}\right. & \left.+X^{H}, Y^{V}+Y^{H}\right) \\
& =\delta p_{i} \wedge d x^{i}\left(X^{i} \delta_{i}+X^{n+i} \partial_{i}, Y^{j} \delta_{j}+Y^{n+j} \partial_{j}\right) \\
& =\delta p_{i}\left(X^{n+i} \partial_{i}\right) \cdot d x^{i}\left(Y^{j} \delta_{j}\right)-\delta p_{i}\left(Y^{n+j} \partial_{j}\right) \cdot d x^{i}\left(X^{i} \delta_{i}\right) \\
& =X^{n+i} Y^{i}-Y^{n+i} X^{i} .
\end{aligned}
$$

On the other hand, the value of $G(P X, Y)$ is

$$
\begin{aligned}
G\left(P\left(X^{V}+X^{H}\right), Y^{V}+Y^{H}\right) & =G\left(X^{V}-X^{H}, Y^{V}+Y^{H}\right) \\
& =G\left(X^{V}, Y^{H}\right)-G\left(X^{H}, Y^{V}\right) \\
& =X^{n+i} Y^{i}-Y^{n+i} X^{i}
\end{aligned}
$$

Therefore, it is seen forward the accuracy of the claim of the theorem.

Theorem 8. Let $M$ be Riemann manifold and let $H$ be Hamiltonian. For any vector field $X$ on $T^{*} M$,

$$
d H(X)=\theta(V, X)
$$

Proof. $d H$ is a 1 -form on $T^{*} M$ with local expression

$$
d H=\frac{\delta H}{\delta x^{i}} d x^{i}+\frac{\partial H}{\partial p_{i}} \delta p_{i}
$$

with respect to adapted local dual frame, and $X$ has local expression

$$
X=X^{j} \frac{\delta}{\delta x^{j}}+X^{n+j} \frac{\partial}{\partial p_{j}}
$$

From (40) and (41), we get

$$
d H(X)=\frac{\delta H}{\delta x^{i}} X^{i}+\frac{\partial H}{\partial p_{i}} X^{n+i}
$$

Since $V$ is a Hamilton vector field, $V$ has local expression with respect to adapted frame on $T^{*} M$ :

$$
V=\frac{d x^{j}}{d t} \frac{\delta}{\delta x^{j}}+\frac{\delta p_{j}}{d t} \frac{\partial}{\partial p_{j}}
$$

where $\delta p_{i} / d t=\left(d p_{i} / d t\right)-p_{k} \Gamma_{i j}^{k}\left(d x^{j} / d t\right)$. Thus we have

$$
\theta(V, X)=G(P V, X)=\frac{d x^{i}}{d t} X^{n+i}-\left(\frac{d p_{i}}{d t}-p_{k} \Gamma_{i j}^{k} \frac{d x^{j}}{d t}\right) X^{i}
$$

If we substitute the above equation into (3), we get

$$
\theta(V, X)=\frac{\partial H}{\partial p_{i}} X^{n+i}+\underbrace{\left(\frac{\partial H}{\partial x^{i}}+p_{k} \Gamma_{i j}^{k} \frac{\partial H}{\partial p_{j}}\right)}_{\delta H / \delta x^{i}} X^{i}
$$

From (43) and (46) it is easily seen that $d H(X)=\theta(V, X)$.

By using the differential geometric objects $H, G, \theta$, and $P$ on the cotangent bundle of the Hamilton space considered in this section, we obtain

$$
d H(X)=\theta(V, X)=G(P V, X)=\nabla_{d \pi(P V)} \omega(d \pi(X)),
$$

where $G$ is the pseudo-Riemannian metric defining the Riemann extension of the Levi-Civita connection on M. If we put $V$ instead of $X$ in the above equation, we obtain

$$
\begin{aligned}
\theta(V, V) & =G(P V, V)=\nabla_{d \pi(P V)} \omega(d \pi(V)) \\
& =d H(V)=V(H)=0 .
\end{aligned}
$$

Since $V(H)=0$, the Hamilton function which gives the total energy of each stage of the system is constant.

\section{The Differential Geometry of $\left(T^{*} M, G\right)$}

In this section, we obtained that the coefficients of the LeviCivita connection $\stackrel{\circ}{\nabla}$ and Riemannian curvature tensor $K$ of $\left(T^{*} M, G\right)$ and we found the condition under which $T^{*} M$ is locally flat.

Theorem 9. The Lie brackets of the horizontal base vector fields $\delta / \delta x^{i}=\partial / \partial x^{i}-N_{i j} \partial / \partial p_{j}, i, j=1, \ldots, n$ and vertical base vector fields $\partial / \partial p_{i}$ on $T^{*} M$ are given by

(i) $\left[\delta / \delta x^{i}, \delta / \delta x^{j}\right]=-R_{k i j} \partial / \partial p_{k}$,

(ii) $\left[\delta / \delta x^{i}, \partial / \partial p_{j}\right]=-\Gamma_{i k}^{j} \partial / \partial p_{k}$,

(iii) $\left[\partial / \partial p_{i}, \partial / \partial p_{j}\right]=0$, where $R_{k i j}=\delta N_{k j} / \delta x^{i}-$ $\delta N_{k i} / \delta x^{j}[2] . R_{k i j}=-p_{h} R_{k i j}^{h}$ for $N_{k i}=-\Gamma_{k i}^{h} p_{h}$.

Theorem 10. Let $(M, H)$ be Hamilton space, $T^{*} M$ the cotangent bundle of $M, G$ a pseudo-Riemann metric defined as Riemann extension of Levi-Civita connection $\nabla$ in $M$, and $\stackrel{\circ}{\nabla}$ the Levi-Civita connection on $T^{*} M$. Then the connection coefficients of the Levi-Civita connection of the pseudo-Riemannian metric $G$ on $T^{*} M$ are given by

$$
\begin{array}{cc}
\stackrel{\circ}{\nabla}_{\delta_{i}} \delta_{j}=-R_{k i j} \partial_{k}, & \stackrel{\circ}{\nabla}_{\delta_{i}} \partial_{j}=-\Gamma_{i k}^{j} \partial_{k}, \\
\stackrel{\circ}{\nabla}_{\partial_{i}} \delta_{j}=\Gamma_{j k}^{i} \partial_{k}, & \stackrel{\circ}{\nabla}_{\partial_{i}} \partial_{j}=0,
\end{array}
$$

where

$$
\delta_{i}=\frac{\delta}{\delta x^{i}}, \quad \partial_{i}=\frac{\partial}{\partial p_{i}}
$$


Proof. Let $X, Y$, and $Z$ be vector fields on $T^{*} M$. According to the Koszul formula, we get

$$
\begin{aligned}
& 2 G\left(\stackrel{\circ}{\nabla}_{X} Y, Z\right) \\
&= X G(Y, Z)+Y G(Z, X)-Z G(X, Y) \\
& \quad-G(X,[Y, Z])-G(Y,[Z, X])+G(Z,[X, Z]) .
\end{aligned}
$$

We put $\delta_{i}, \delta_{j}$, and $\delta_{k}$ instead of $X, Y$, and $Z$ in (51); then we get

$$
2 G\left(\stackrel{\circ}{\nabla}_{\delta_{i}} \delta_{j}, \delta_{k}\right)=R_{i j k}+R_{j k i}-R_{k i j}
$$

By the equality $\sum_{(i, j k)} R_{i j k}=0$, we find

$$
G\left(\stackrel{\circ}{\nabla_{\delta_{i}}} \delta_{j}, \delta_{k}\right)=-R_{k i j}
$$

and we put $\delta_{i}, \delta_{j}$, and $\partial_{k}$ instead of $X, Y$, and $Z$ in (51). So we get

$$
2 G\left(\stackrel{\circ}{\nabla}_{\delta_{i}} \delta_{j}, \partial_{k}\right)=\Gamma_{j i}^{k}-\Gamma_{i j}^{k}
$$

Since the Levi-Civita connection $\nabla$ which is defined on $M$ is torsion-free, we have $\Gamma_{j i}^{k}=\Gamma_{i j}^{k}$. Subsequently we find

$$
G\left(\stackrel{\circ}{\nabla}_{\delta_{i}} \delta_{j}, \partial_{k}\right)=0
$$

Thus we get

$$
\stackrel{\circ}{\nabla}_{\delta_{i}} \delta_{j}=-R_{k i j} \partial_{k}
$$

and the rest of the equalities can be obtained similarly.

Theorem 11. Let $M$ be a Hamilton space, $T^{*} M$ the cotangent bundle of $M, G$ a pseudo-Riemann metric defined as Riemann extension of Levi-Civita connection $\nabla$ in $M, \stackrel{\circ}{\nabla}$ the Levi-Civita connection on $T^{*} M$, and $K$ the Riemann curvature tensor on $T^{*} M$. Then the components of the Riemann curvature tensor on $T^{*} M$ are given by

$$
\begin{gathered}
K\left(\delta_{i}, \delta_{j}\right) \delta_{k} \\
=\left(-\delta_{i} R_{h j k}+\delta_{j} R_{h i k}+R_{l j k} \Gamma_{i h}^{l}-R_{l i k} \Gamma_{j h}^{l}+R_{l i j} \Gamma_{k h}^{l}\right) \partial_{h}, \\
K\left(\delta_{i}, \delta_{j}\right) \partial_{k}=\left(\partial_{k} R_{h i j}\right) \partial_{h}, \\
K\left(\delta_{i}, \partial_{j}\right) \delta_{k}=\delta_{k}\left(\Gamma_{i h}^{j} \circ \pi\right) \partial_{h}, \\
K\left(\partial_{i}, \delta_{j}\right) \delta_{k}=-\delta_{k}\left(\Gamma_{j h}^{i} \circ \pi\right) \partial_{h}, \\
K\left(\delta_{i}, \partial_{j}\right) \partial_{k} \quad \\
=K\left(\partial_{i}, \delta_{j}\right) \partial_{k}=K\left(\partial_{i}, \partial_{j}\right) \delta_{k}=K\left(\partial_{i}, \partial_{j}\right) \partial_{k}=0 .
\end{gathered}
$$

Proof. Let $X, Y$, and $Z$ be vector fields on $T^{*} M$. Then

$$
K(X, Y,) Z=\stackrel{\circ}{\nabla}_{X} \stackrel{\circ}{\nabla}_{Y} Z-\stackrel{\circ}{\nabla}_{Y} \stackrel{\circ}{\nabla}_{X} Z-\stackrel{\circ}{\nabla}_{[X, Y]} Z
$$

If we put $\delta_{i}, \delta_{j}$, and $\partial_{k}$ instead of $X, Y$, and $Z$ in (58), we get

$$
\begin{aligned}
K\left(\delta_{i}, \delta_{j}\right) \partial_{k}= & \stackrel{\circ}{\nabla}_{\delta_{i}} \stackrel{\circ}{\nabla}_{\delta_{j}} \partial_{k}-\stackrel{\circ}{\nabla}_{\delta_{j}} \stackrel{\circ}{\nabla}_{\delta_{i}} \partial_{k}-\stackrel{\circ}{\nabla}_{[\underbrace{}_{\epsilon V T^{*} M}, \delta_{j}]}^{\partial_{k}} \\
= & -\delta_{i}\left(\Gamma_{j l}^{k} \circ \pi\right) \partial_{l}+\delta_{j}\left(\Gamma_{i l}^{k} \circ \pi\right) \partial_{l} \\
& -\Gamma_{j l}^{k} \Gamma_{i h}^{l} \partial_{h}+\Gamma_{i l}^{k} \Gamma_{j h}^{l} \partial_{h} \\
= & \left(-\frac{\partial \Gamma_{j h}^{k}}{\partial x^{i}}+\frac{\partial \Gamma_{i h}^{k}}{\partial x^{j}}-\Gamma_{j l}^{k} \Gamma_{i h}^{l}+\Gamma_{i l}^{k} \Gamma_{j h}^{l}\right) \partial_{h} \\
= & -R_{h i j}^{k} \partial_{h} .
\end{aligned}
$$

By the equality $R_{h i j}=-p_{k} R_{h i j}^{k}$, we obtain

$$
K\left(\delta_{i}, \delta_{j}\right) \partial_{k}=\frac{\partial R_{h i j}}{\partial p_{k}} \partial_{h} .
$$

The other coefficients of the curvature tensor can be obtained similarly.

Theorem 12. The pseudo-Riemann manifold $\left(T^{*} M, G\right)$ is flat if and only if the Riemann manifold $(M, g)$ is Euclidean.

Proof. If the Riemann manifold $(M, g)$ is Euclidean, the Christoffel symbols must be zero. Thus, Riemann curvature tensor $R$ on $M$ and $K$ on $T^{*} M$ must be zero.

\section{Concluding Remarks}

The projected curves in the Hamilton space of the integral curves of the Hamilton vector field are geodesics. Furthermore, the total energy of each stage of the system is constant. The cotangent bundle of the Hamilton space is flat if and only if the Hamilton space is Euclidean.

\section{References}

[1] R. Miron, The Geometry of Higher-Order Hamilton Spaces Applications to Hamiltonian Mechanics, Kluwer Academic, Dordrecht, The Netherlands, 2003.

[2] V. Oproiu and N. Papaghiuc, "A pseudo-Riemannian structure on the cotangent bundle," Analele Ştiințifice ale Universităţii Al. I. Cuza din Iaşi. Serie Nouă. Matematică, vol. 36, no. 3, pp. 265276, 1990.

[3] T. Willmore, "Riemann extensions and affine differential geometry," Results in Mathematics, vol. 13, no. 3-4, pp. 403-408, 1988.

[4] K. Yano and S. Ishihara, Tangent and Cotangent Bundles, Marcel Dekker, New York, NY, USA, 1973.

[5] S. Akbulut, M. Özdemir, and A. A. Salimov, "Diagonal lift in the cotangent bundle and its applications," Turkish Journal of Mathematics, vol. 25, no. 4, pp. 491-502, 2001. 
[6] V. Oproiu, "A pseudo-Riemannian structure in Lagrange geometry," Analele Ştiințifice ale Universității Al. I. Cuza din Iaşi. Serie Nouă. Secţiunea I, vol. 33, no. 3, pp. 239-254, 1987.

[7] I. Ayhan, "Lifts from a Lagrange manifold to its contangent bundle," Algebras, Groups and Geometries, vol. 27, no. 2, pp. 229246, 2010.

[8] I. Ayhan, "L-dual lifted tensor fields between the tangent and cotangent bundles of a Lagrange manifold," International Journal of Physical and Mathematical Sciences, vol. 4, no. 1, pp. 86-93, 2013.

[9] A. Polnarev, Relativity and Gravitation, vol. 5 of Lecture Notes, 2010.

[10] R. Miron, H. Hrimiuc, H. Shimada, and V. S. Sabau, The Geometry of Hamilton and Lagrange Spaces, Kluwer Academic, New York, NY, USA, 2001.

[11] V. I. Arnold, Mathematical Methods of Classical Mechanics, Springer, Berlin, Germany, 1989.

[12] R. Abraham and J. E. Marsden, Foundations of Mechanics, W. A. Benjamin, New York, NY, USA, 1967. 


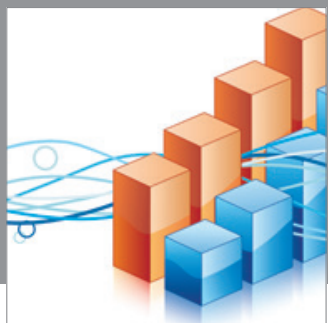

Advances in

Operations Research

mansans

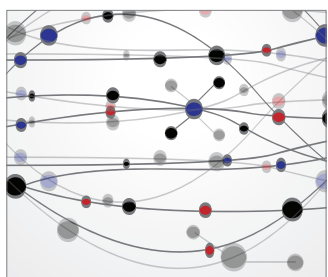

The Scientific World Journal
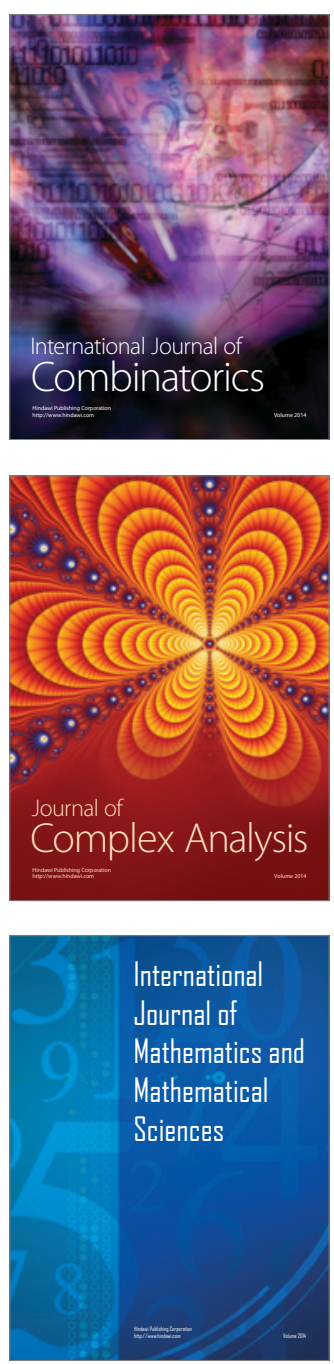
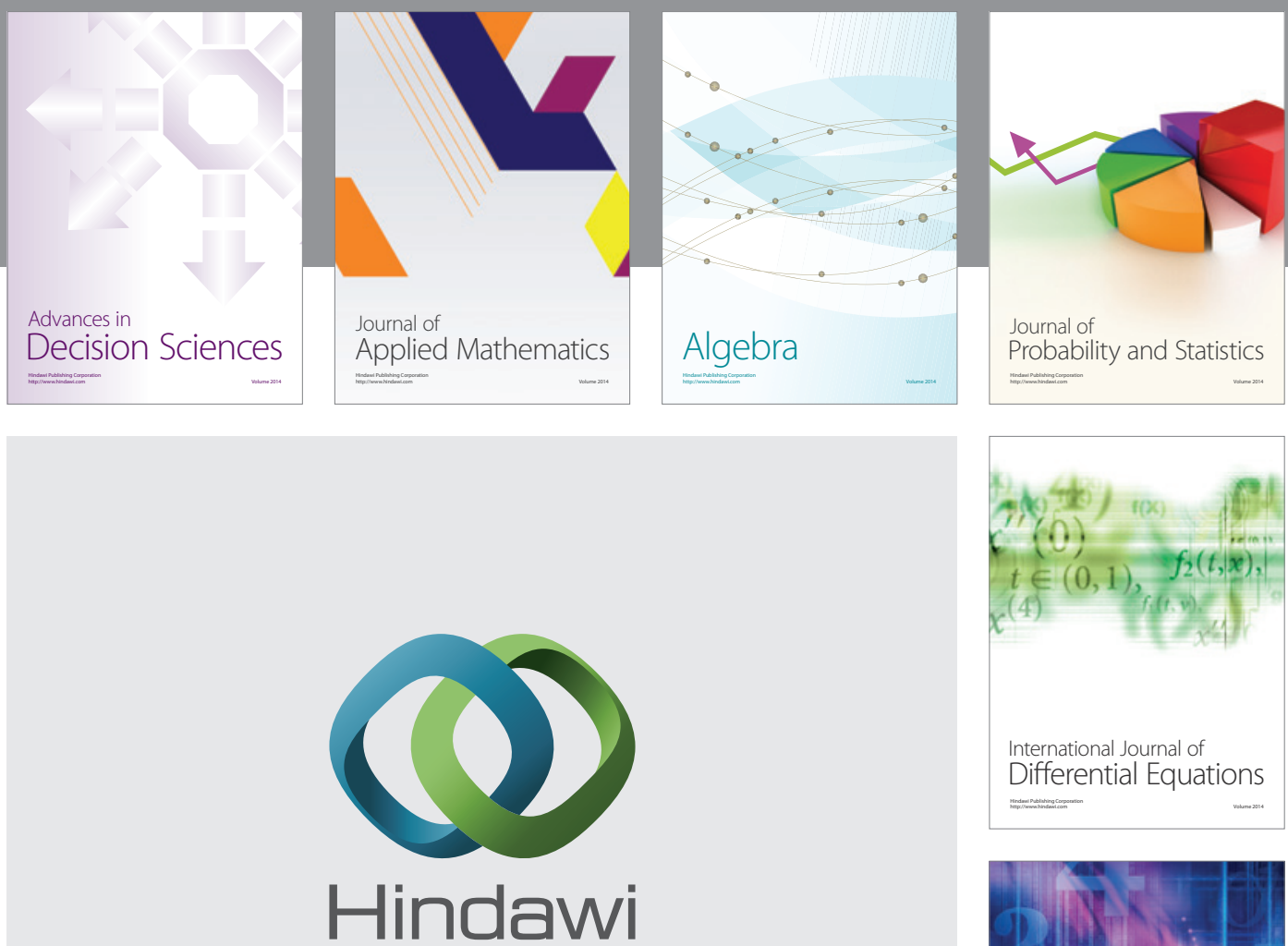

Submit your manuscripts at http://www.hindawi.com
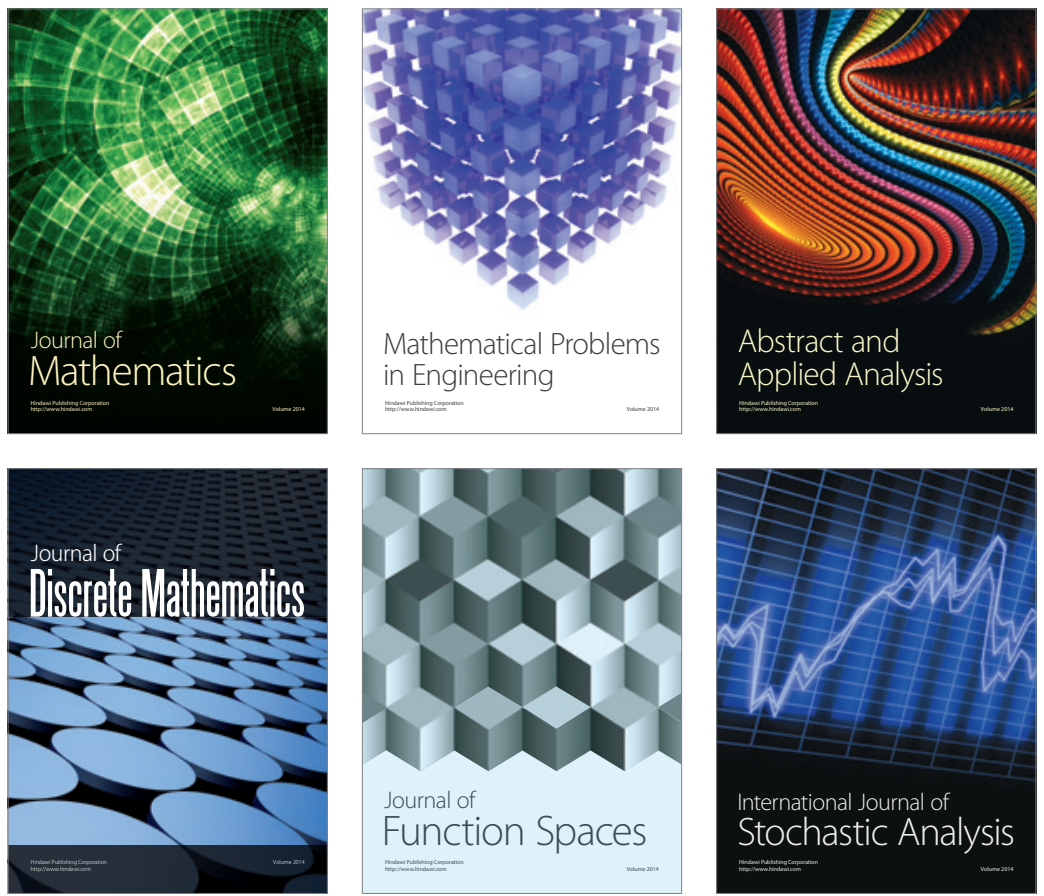

Journal of

Function Spaces

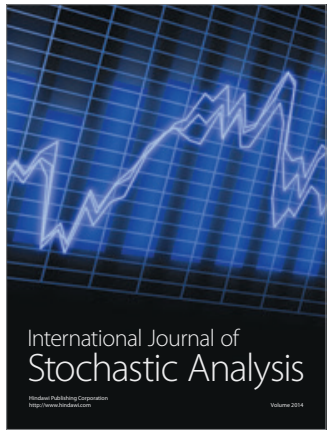

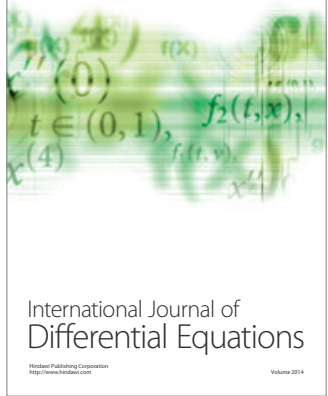
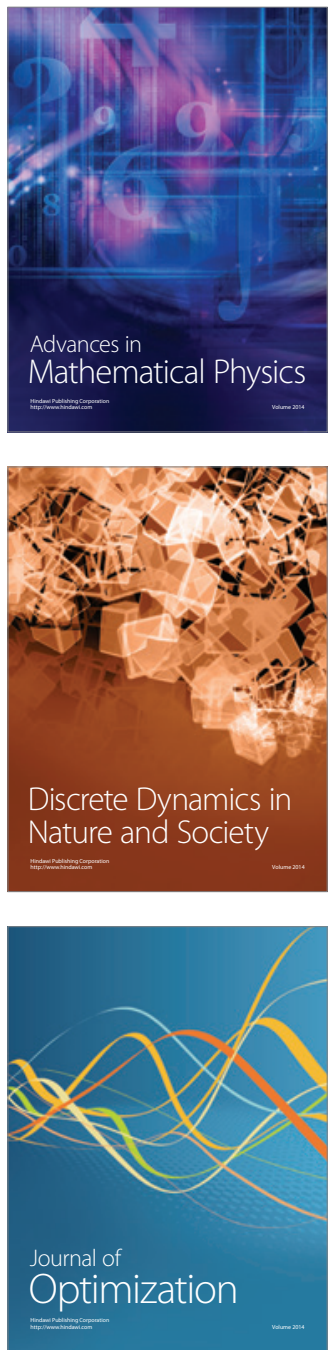